\title{
Traitement de la paille de blé à l'ammoniac et à l'urée : effet sur la digestibilité ruminale et sur le comportement alimentaire et mérycique chez les bovins
}

\author{
A Chermiti 2, E Teller ${ }^{1}$, B Matatu ${ }^{1}$, M Vanbelle 1 \\ 1 UCL, laboratoire de Biochimie de la nutrition, Louvain-la-Neuve, Belgique; \\ 2 INRAT, laboratoire de Nutrition animale, Ariana 2049, Tunisie
}

\begin{abstract}
Summary - Ammonia and urea treatment of wheat straw: effect on ruminal digestibility and chewing behaviour in cattle. Three rumen and duodenum cannulated cows were fed $6 \mathrm{~kg}$ DM of untreated or ammonia- or urea-treated wheat straw supplemented with $2.6 \mathrm{~kg} \mathrm{DM}$ of concentrate mixture. Ruminal OM digestibility of the 3 diets was similar, whereas the duodenal flow of $\mathrm{N}$ was increased with treated straws. Chewing time was reduced after treatment owing to the higher rate of intake and the reduced ruminating time.
\end{abstract}

L'objectif de cette étude est d'examiner l'effet du traitement de la paille à l'ammoniac ou à l'urée sur la digestibilité ruminale, le flux d'azote duodénal et le comportement alimentaire des bovins.

Matériel et méthodes - Deux meules $(1700$ $\mathrm{kg}$ ) de paille de froment (variété Camp Remy) ont été traitées à la dose de $30 \mathrm{~g}$ de $\mathrm{NH}_{3}$ ou de $53 \mathrm{~g}$ de $\mathrm{CO}\left(\mathrm{NH}_{2}\right)_{2} / \mathrm{kg}$ de MS. Après conservation sous plastique pendant 5 sem et hachage $(3 \mathrm{~cm})$, la paille non traitée (PNT) et celles traitées à l'ammoniac (PTA) ou à l'urée (PTU) ont été distribuées (6 $\mathrm{kg} \mathrm{MS} / \mathrm{j})$ à 3 vaches taries, pesant $575 \mathrm{~kg}$, au cours de 3 périodes de $38 \mathrm{j}$. Le concentré $(2,6 \mathrm{~kg}$ $\mathrm{MS} / \mathrm{j}$ ) était composé de $70 \%$ d'orge, $20 \%$ de pulpe de betterave et de $10 \%$ de soja; il contenait $175 \mathrm{~g}$ d'urée avec la ration PNT. Le $\mathrm{Cr}_{2} \mathrm{O}_{3}(20 \mathrm{~g})$ et le PEG (90 g) ont été introduits en 2 fois ( $8 \mathrm{~h}$ et $16 \mathrm{~h}$ ) par la canule ruminale pour mesurer les flux des digesta duodénaux. Les prélèvements du contenu duodénal $(300 \mathrm{~g})$ ont été effectués en 3 périodes réparties sur $2 \mathrm{j}$ successifs : de $7 \mathrm{~h}$ à $13 \mathrm{~h}$ le premier jour, de $23 \mathrm{~h}$ à $5 \mathrm{~h}$ et de $15 \mathrm{~h}$ à $21 \mathrm{~h}$ le deuxième jour. Les échantillons ont été cumulés pour chaque vache et chaque période pour des analyses ultérieures. Le flux duodénal a été calculé sur la base de la concentration des 2 marqueurs (Teller et al, 1989). L'azote d'origine bactérienne a été estimé à partir du dosage de l'acide diaminopimélique. Le comportement alimentaire et mérycique a été enregistré selon la technique de Beauchemin et al (1989). Les déterminations de MS, des cendres, de l'azote et du NDF ont porté sur des échantillons de contenu duodénal lyophilisé (Teller et al, 1989) et les résultats ont été soumis à une analyse de la variance.

Résultats et discussion - Bien que le traitement ait augmenté la teneur en $\mathrm{N}$ de la paille de 4,7 à $20 \mathrm{~g} / \mathrm{kg}$ de $\mathrm{MS}$, la digestibilité ruminale de la $\mathrm{MO}$ des 3 rations était quasiment identique. L'effet du traitement apparaît au niveau du flux d'azote duodénal qui était augmenté d'environ 20\% (tableau I). Cette augmentation s'explique essentiellement par le niveau supérieur du flux d'azote bactérien obtenu avec les régimes de pailles traitées (104 contre $81 \mathrm{~g}$ ). La durée d'ingestion par $\mathrm{kg}$ de MS de PTA ou de PTU est de 52 et $60 \mathrm{~min}$, respectivement, alors qu'elle est de 74 min pour la PNT. L'augmentation de la fragilité de la paille suite aux traitements (Zorrilla-Rios et al, 1985) est en partie à l'origine de cette différence.

En conclusion, le traitement de la paille à l'ammoniac ou à l'urée a eu un impact relativement faible sur la digestibilité ruminale de la MO en comparaison à l'amélioration observée chez des moutons recevant les mêmes rations (Chermiti et al, non publié). Le flux de matières azotées totales arri- 
vant dans l'intestin est nettement augmenté avec les régimes pailles traitées. La durée de mastication des pailles traitées est réduite d'environ $10 \%$, ce qui est le résultat de l'ingestion plus rapide et du temps de rumination plus court.
Beauchemin KA, Zelin S, Genner D, BuchananSmith JG (1989) J Dairy Sci 72, 2746-2759

Teller $E$, Vanbelle $M$, Kamatali $P$, Wavreille $J$ (1989) J Anim Sci 67, 2802-2809

Zorrilla-Rios J, Owens FN, Horn GW (1985) JAnim Sci 60, 814-821

Tableau I. Effets du traitement de la paille sur la digestibilité ruminale de la ration totale, le flux d'azote duodénal et le comportement alimentaire et mérycique chez les bovins.

\begin{tabular}{|c|c|c|c|c|}
\hline & PNT & PTA & PTU & $E S M$ \\
\hline \multicolumn{5}{|l|}{ Ingestion $(g / j)$} \\
\hline MS & 8403 & 8912 & 8633 & 225 \\
\hline NDF & 5904 & 6474 & 6091 & 150 \\
\hline$N$ & 180 & 185 & 184 & 2 \\
\hline \multicolumn{5}{|c|}{ Digestibilité ruminale de la ration (\%) } \\
\hline MO 1 & 51,6 & 54,3 & 55,1 & 1,4 \\
\hline NDF & 63,7 & 71,3 & 68,1 & 1,6 \\
\hline \multicolumn{5}{|l|}{ Flux duodénal $(g / j)$} \\
\hline $\mathrm{N}$ & 148 & 185 & 176 & 18 \\
\hline $\mathrm{N}$ bactérien & 81 & 104 & 104 & 12 \\
\hline \multicolumn{5}{|l|}{ Durée d'ingestion } \\
\hline $\min / \mathrm{j}$ & 422 & 323 & 362 & 6 \\
\hline min/kg MS de paille ingérée & 74 & 52 & 60 & 1 \\
\hline \multicolumn{5}{|l|}{ Durée de rumination } \\
\hline $\min / j$ & 490 & 475 & 458 & 9 \\
\hline $\mathrm{min} / \mathrm{kg}$ MS de paille ingérée & 58 & 53 & 53 & 1 \\
\hline
\end{tabular}

' Corrigée pour la contribution bactérienne dans le contenu duodénal. 